\title{
The effect of the liquid layer thickness on the evaporation intensity
}

\author{
Aleksei $\mathrm{Kreta}^{1,2,3^{*}}$, and Vyacheslav Maksimov ${ }^{3}$ \\ ${ }^{1}$ Novosibirsk State University, 630090 Novosibirsk, Russia \\ ${ }^{2}$ Kutateladze Institute of Thermophysics, 630090 Novosibirsk, Russia \\ ${ }^{3}$ National Research Tomsk Polytechnic University, 634050 Tomsk, Russia
}

\begin{abstract}
An experimental study of the influence of thermo-capillary forces and shear stresses with the side of the gas flow to the evaporation flow rate has been made. The experiments were carried out at various thicknesses of the liquid layer and constant gas velocity. The influence of the thickness of the liquid layer on the evaporation flow rate (the intensity of evaporation) has been analyzed. It is shown that the thermocapillary forces have a direct effect on the evaporation flow rate of the liquid layer.
\end{abstract}

\section{Introduction}

One of the main directions of modern technology development is the miniaturization of electronic equipment [1]. As a result, with decreasing the size of electronic devices, the specific and volumetric density of the heat flux increases greatly. For removing high heat fluxes, promising two-phase cooling system in which a thin layer of liquid moves in a flat mini or micro channel under the action of a gas stream is used. In such systems, the relationship between evaporation and convection in the liquid layer has a direct effect on heat and mass transfer. Intensive evaporation from the free surface of a liquid under the action of a gas flow causes different convection currents in the liquid layer. The shape and structure of these flows are quite complex and can have a significant effect on the intensity of evaporation and, consequently, on the efficiency of heat exchangers.

Convective flows of liquids, accompanied by evaporation at the interface, are actively studied experimentally and theoretically [2-6]. The space experiment "Evaporative convection and interphase heat and mass transfer" is devoted to the study of thermocapillary convection in a liquid layer due to intensive evaporation under the action of a gas flow [2]. This is a joint experiment of the European Space Agency and Russian Space Agency, which will be planned to carry out on aboard the International Space Station. Numerical study of the development of convective currents in horizontal liquid layers of different thickness evaporating under the action of a gas flow was carried out in [4]. It is shown that in time both the cross-section and longitudinal dimensions of the vortex structures increase. Also it is shown that an increase in the thickness of the liquid layer also contributes to the growth of the dimensions of the vortex structures. The development of mathematical models of

\footnotetext{
* Corresponding author: alexy.slav@yandex.ru
} 
convective motions with allowance for the processes of heat and mass transfer at the interface and the formulation of conditions on the interface on the basis of exact solutions of the Navier-Stokes equations in the Oberbeck-Boussinesq approximation are reviewed in $[5,7]$. In article [7], a comparison of the analytical and experimental results [2] on the evaporation of liquid from the thermocapillary interface was carried out and their qualitative coincidence was revealed.

The aim of the work is an experimental study of the interaction of various mechanisms of convective currents arising due to thermogravitational, thermocapillary convection, as well as shear stresses, on the intensity of evaporation of a horizontal moving liquid layer into a gas stream.

\section{Experimental setup}

The experimental investigations were conducted on the setup shown in Fig. 1. The setup consists of the following components: test section, gas and liquid loops, data acquisition system, thermal stabilization system, and optical techniques.

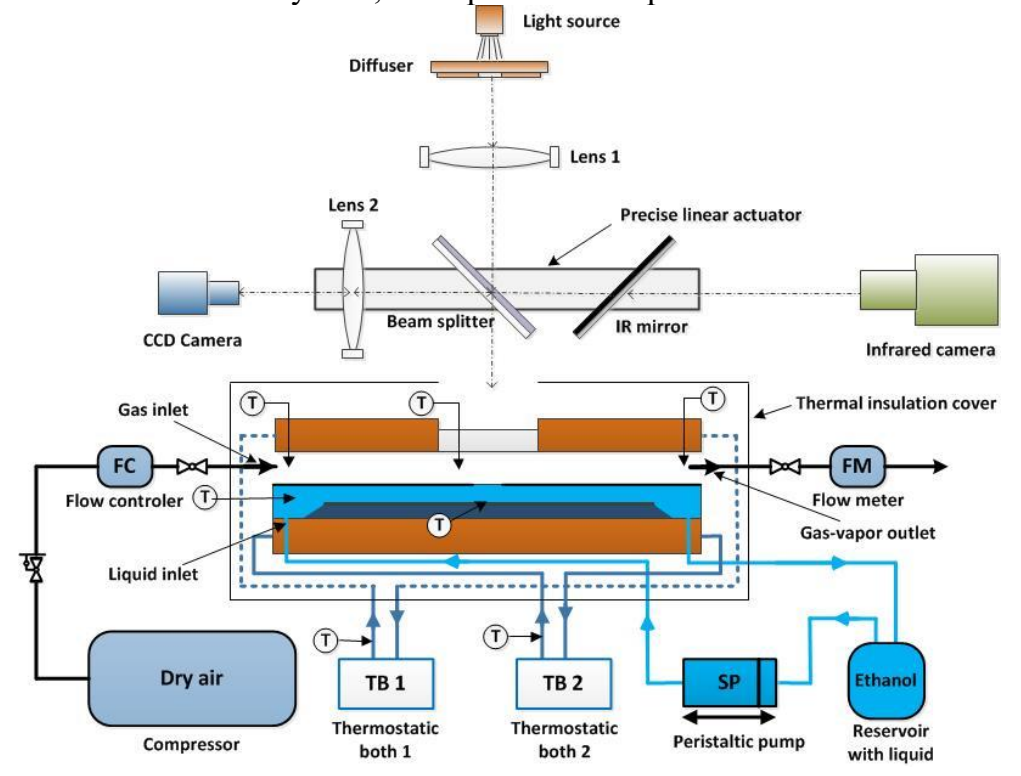

Fig. 1. Scheme of the experimental setup.

In the centre of the installation there is a working section. The working area consists of gas and liquid channels, which are separated by a thin metal plate having a thickness of 200 microns. The metal plate has a square cut-out in the centre of $10 \times 10 \mathrm{~mm}$. It is at this point that the liquid contacts the gas. The height of the liquid channel is changed by means of metal plates. Thermostabilization of the working section was carried out by two thermostats TB1 and TB2. Gas enters to the test section from the compressor. The gas flow rate at the inlet of the gas channel was set by means of a mass flow regulator. The liquid was injected into the liquid channel by a peristaltic pump. The liquid flow rate in the channel was changed by changing the flow rate at the peristaltic pump. The working fluid evaporated under the action of the gas flow and the vapor-gas flow moved to the outlet of the gas channel of the working section. The flow of the vapor-gas mixture at the outlet of the working section was measured with a mass flow meter. The position of the interface level was controlled with an accuracy of 10 micrometres using a Schlieren technique and the peristaltic pump. 
According to the experimental conditions, the interface between the liquid and the gas must be maintained in a flat state throughout the entire experiment. The position control of the level of the interphase boundary was carried out using the method Schlieren and the peristaltic pump. At the same time, it was necessary to measure the temperature distribution on the interphase surface. Measurements of the temperature distribution on gas-liquid interface were conducted with the help of Titanium 570M IR camera. Infrared imaging of liquid surface was performing through quartz optical window that implemented in the top cover of the test cell. IR camera was placed on the optical tabletop horizontally and measuring of the surface temperature of the liquid was contacted with the help of the IR mirror. The IR mirror was inclined to 450 to infrared camera and liquid surface. Optical components of the Schlieren technique and IR mirror were mounted on an optical platform of the linear actuator. Linear actuator is controlled from personal computer and by special software. The optical platform moved in the range of $50 \mathrm{~mm}$ and speed of $104 \mathrm{~mm} / \mathrm{s}$ in horizontal direction. Initially, the platform was set in position where optical axes of Schlieren technique coincide and the interface visualized. When a flatness of interface and the stationary regime of the experiment were established the linear actuator moved the platform in position where optical axes of the IR camera and mirror also coincide and measurement of surface temperature was running.

\subsection{Calculation of shear stresses}

Fig. 2 shows a thermogram of the liquid surface at the intensive evaporation. The IR image snapshot does not explain all dynamic of the process. Analyzing of the video the movement of gas-liquid interface in counter-current direction to the gas flow is observed.

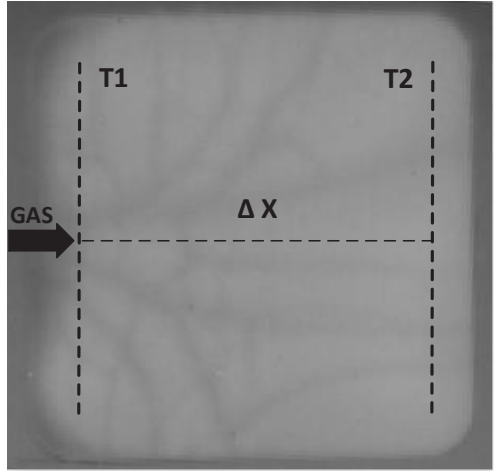

Fig. 2. The thermogram of the evaporating liquid layer.

Convective flows in the liquid layer propagate under the action of thermocapillary forces and shearing stresses caused by friction of the gas flow on the interphase surface. Thermocapillary stresses on the surface of the liquid layer caused by the action of the thermocapillary effect are calculated by the formula:

$$
\tau_{s u r}=\frac{\delta \sigma}{\delta T} \frac{\delta T}{\delta x}
$$

where, $\sigma_{T}=\frac{\delta \sigma}{\delta T}$ - temperature coefficient of surface tension is equal $1.14 \times 10^{-4} \mathrm{~N} / \mathrm{mK}$.

The temperature gradient is defined as: $\frac{\delta T}{\delta x}=\frac{T_{2}-T_{1}}{\Delta x}$. Points of the temperature measuring and length $x=0.008 \mathrm{~m}$ are shown in Fig. 2 . 
Shear stresses on the liquid surface induced by gas flow can be estimated as [8]:

$$
\tau_{g}=\eta \frac{d V}{d y}=-\frac{H}{2} \frac{\delta p}{\delta x}
$$

where, $H=0.005 \mathrm{~m}$ is height of gas channel; $\eta$-dynamic viscosity.

Pressure gradient is calculated from the formula: $V_{g}=-\frac{H^{2}}{12 \eta} \frac{\delta P}{\delta x}$, where average gas velocity $V_{g}, \mathrm{~m} / \mathrm{c}$ is known value. It is assumed that in the gas channel the Poiseuille flow is realised. The gas-liquid interface is considered as a rigid surface. In more detail, the method of calculating tangential stresses is described in the article [9].

\section{Results and discussion}

The experiments were conducted at atmospheric pressure in a test section. The thickness of the liquid layer in the experiments was 3,5 and $8 \mathrm{~mm}$, respectively. Ethanol liquid was used as the working liquid. The evaporation surface area was $100 \mathrm{~mm}^{2}$ with the corresponding $10 \times 10 \mathrm{~mm}$ cut-out in the plate. The temperature of the "liquid-gas" system has changed from $20{ }^{\circ} \mathrm{C}$ to $40^{\circ} \mathrm{C}$, with the step of $10^{\circ} \mathrm{C}$. The difference between the temperature of the gas and liquid does not exceed $0.1{ }^{\circ} \mathrm{C}$. The gas flow rate was a constant and equaled $1000 \mathrm{ml} / \mathrm{min}$ that corresponds to gas average velocities $0.138 \mathrm{~m} / \mathrm{s}$. Reynolds fluid throughout the experiment was constant and was equal to $10(\mathrm{Re}=10)$.

Dependency of the evaporation flow rate on the depth of the liquid layer at different temperatures is shown in Fig. 3

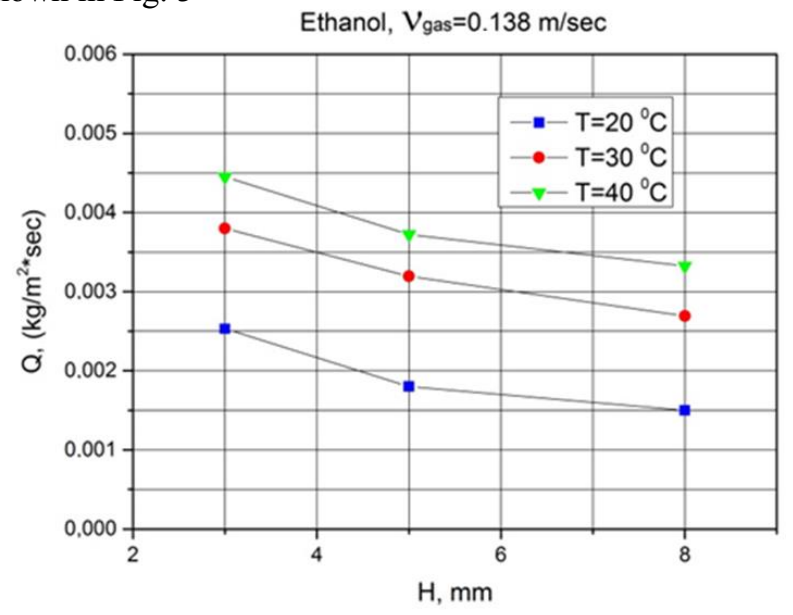

Fig. 3. Evaporation flow rate.

It can be seen from the graph that the maximum evaporation rate is reached with a liquid layer thickness equal to $3 \mathrm{~mm}$. With increasing thickness of the liquid layer, the evaporation flow rate decreases substantially. On average, the evaporation rate decreases by $30 \%$. It is shown that this recession is caused by a sharp drop in thermocapillary forces on the interphase surface of the phase section. It is supposed that this is connected with the complication of the structure of convective currents in the liquid layer with increasing thickness. Using a thermal imager, it was noted that as the thickness of the layer increases, the structure of the flow in the liquid layer changes, which directly affects the evaporation flux rate. With increasing thickness of the liquid layer, the number of convective structures in the liquid layer increases, 
and as a result, the mass flow through the interfacial surface decreases. A similar phenomenon in the case of a stationary liquid layer was observed by Lyulin and Kabov [2].

Fig. 4 shows the dependence of the thermocapillary stresses on the thickness of the liquid layer at various temperatures of the "liquid-gas" system.

Ethanol, $V_{\text {gas }}=0.138 \mathrm{~m} / \mathrm{sec}$

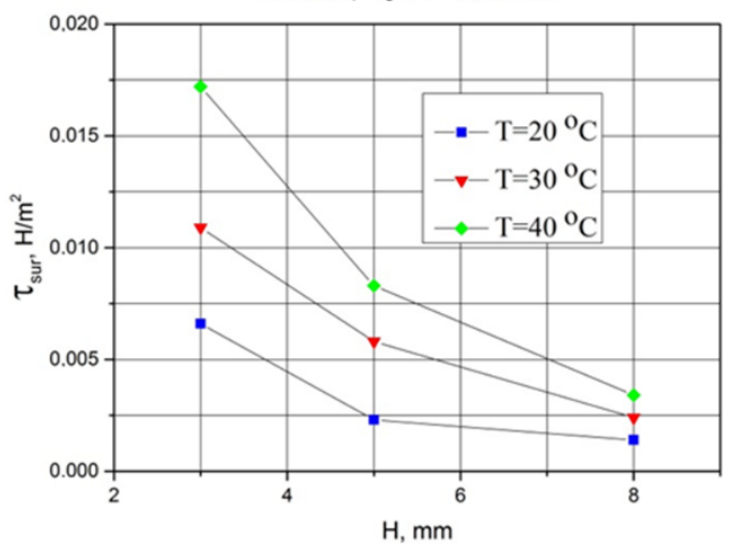

Fig. 4. Thermocapillary stresses.

It can be seen from the graph that the thermocapillary forces on the interphase surface decrease significantly with increasing thickness of the liquid layer. First of all, this drop is due to the fact that the influence of gravitational forces increases with increasing layer of liquid. The influence of gravitational forces significantly reduces the temperature gradient on the interphase surface, which leads to a sharp decrease in thermocapillary forces. Secondly, as already mentioned, when the thickness of the liquid layer is increased, the structure of the convective currents in the layer becomes much more complicated, which also leads to a drop in the gradient of the temperature on the interphase surface. Such a drop in thermocapillary forces has a direct effect on the rate of evaporation.

Ethanol, $V_{\text {gas }}=0.138 \mathrm{~m} / \mathrm{sec}$

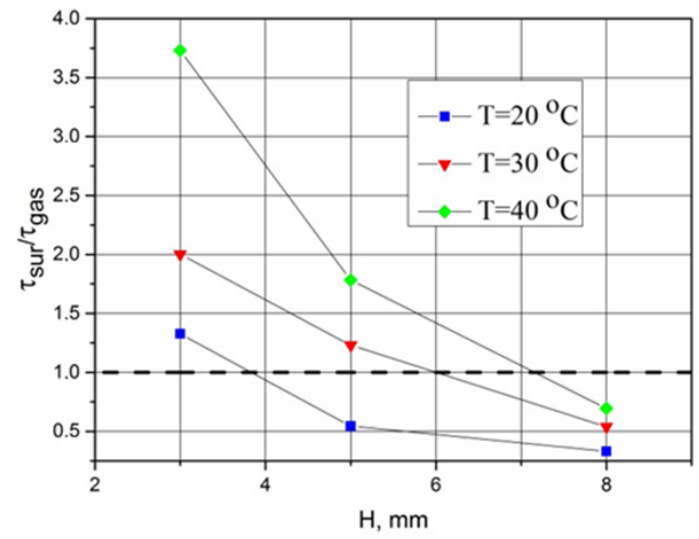

Fig. 5. The ratio of shear stresses.

It was also noted that with increasing thickness of the layer, the thermo-capillary shear stress to the gas shear stress are decreased (Fig. 5). With a maximum layer thickness in the experiment of $8 \mathrm{~mm}$ for all diapasons of the "liquid-gas" temperatures, the shear stresses on the gas flow side are greater than the thermocapillary stresses. This correlation of forces directly affects the character and rate of development of convective currents in the liquid layer and, as a result, on the intensity of evaporation. 


\section{Conclusions}

Experiments were conducted to study the influence of the liquid layer thickness on the evaporation flow rate at various temperatures of the "liquid-gas" system and at a constant gas flow rate. Infrared study and measurements of the temperature field on the surface of horizontal liquid layer evaporating into gas flow has been performed. Temperature gradient of the gas-liquid interface has been measured with the help of Titanium 570M IR camera. Shear stresses on gas-liquid interface induced by thermocapillary effect and inert gas flow have been defined. It is shown that the thermocapillary stresses have a direct effect on the evaporation flow rate of the liquid layer. It is found that as the thickness of the liquid layer increases, the evaporation intensity decreases as a result of a sharp decrease in thermocapillary forces on the interphase surface.

The work was financially supported by Russian Foundation for Basic Research (Project identifier No. 17-38-50223 “mol_nr”).

\section{References}

1. E. A. Chinnov, V. V. Guzanov, O. A. Kabov, Letters to the Journal of Technical Physics 35, 14 (2009)

2. Yu.V. Lyulin, O. A. Kabov, Int. J. Heat Mass Transf. 70, 599 (2014)

3. B. Scheid, J. Margerit, C. S. Iorio, L. Joannes, M. Heraud, P. Queeckers, P. C. Dauby, P. Colinet, Experiments in Fluids 52, 1107 (2012)

4. H. Machrafi, C. S. Iorio, P. C. Dauby, Interfacial Phenomena and Heat Transfer 2, 3 (2014)

5. N. A. Goncharova, O. A. Kabov, Int. J. Heat Mass Transf. 53, 2795 (2010)

6. N. A. Goncharova, M. Hennenberg, E. V. Rezanova, O. A. Kabov, Interfacial phenomena and heat transfer 1, 3 (2013)

7. N. A. Goncharova, E. V. Rezanova, Yu. V. Lyulin, O. A. Kabov, Thermophys. Aeromech. 22, 5 (2015)

8. L. Landau, E. Lifshitz, Hydrodynamics (Science, Moscow, 1986)

9. A. S Kreta, Yu. V. Lyulin, MATEC Web Conf. 92, 01048 (2017) 